\title{
PERCUTANEOUS EPIDIDYMAL SPERM ASPIRATION (PESA) IN MEN WITH OBSTRUCTIVE AZOOSPERMIA
}

\author{
SIDNEY GLINA, JORGE B. FRAGOSO, FERNANDO G. MARTINS, JONATHAS B. SOARES, \\ ANDREA G. GALUPPO, ROBERTA WONCHOCKIER
}

\author{
Human Reproduction Unit, Albert Einstein Jewish Hospital, São Paulo, SP, Brazil
}

\begin{abstract}
Objectives: Assessing the efficiency of repeated percutaneous epididymal sperm aspiration (PESA) in men with obstructive azoospermia, and also the possibility of cryopreservation of remaining material for future use in intracytoplasmic sperm injection (ICSI).

Method: Retrospective study, in which 79 procedures of PESA were assessed in 58 patients (mean age $=45$ years), whose partners had mean age of 34 years. Vasectomy was the most frequent cause of obstructive azoospermia $(n=46)$.

Results: Motile spermatozoa were obtained in 65 procedures (82\%). PESA was twice repeated for 15 patients, 3 times for 5 patients, and 4 times for 1 patient. Spermatozoa were found in $13(87 \%)$ patients in the second attempt, in $4(80 \%)$ patients in the third attempt, and in the only patient that had accomplished 4 procedures. In 30 procedures (37\%), we have obtained enough material for cryopreservation. In 12 among the 13 samples thawed ( $\mathrm{n}=13$ patients), motile spermatozoa were found, and ICSI was accomplished. Four patients that did not use their samples requested the elimination of the material. Total rate of pregnancy per transference was 21/55 (38\%). In 14 PESA procedures, it was not possible to find spermatozoa; in these cases, the patients opted for accomplishing the procedure of testicular sperm aspiration (TESA).

Conclusion: PESA is an efficient and simple method of retrieving spermatozoa, allowing repeated procedures. Additionally, spermatozoa collected through PESA can be cryopreserved.
\end{abstract}

Key words: male infertility; azoospermia; sperm; aspiration; epididymis; testis; punctures Int Braz J Urol. 2003; 29: 141-6

\section{INTRODUCTION}

The use of intracytoplasmic sperm injection (ICSI) as clinical practice revolutionized the treatment of patients with severe male factor of infertility (1). Even patients bearing obstructive azoospermia can benefit from the ICSI technique to obtain pregnancy, by collecting spermatozoa directly from the epididymis. The first method to be introduced was the microsurgical aspiration of spermatozoa from the epididymis (MESA - microscopic epididymal sperm aspiration) in 1985 (2,3), followed in 1995 by the development of another method, percutaneous epid- idymal sperm aspiration (PESA) $(3,4)$. Since then, both techniques have been constantly compared in relation to efficiency, easiness of execution, patient recovery, possibility of repetition for the same patient, among other factors.

Initially, spermatozoa obtained from the epididymis were used in in-vitro fertilization (FIV), but rates of fertilization and pregnancy by transference were low, as for instance with values of $11 \%$ for fertilization rate and $8 \%$ for pregnancy rate $(2,5,6)$. With the development of ICSI, results obtained became to be better, showing that the 2 techniques (PESA and ICSI) can be used together and successfully (4). In a 
work described by Friedler et al., fertilization rate of $56 \%$ and pregnancy rate by transference of $31 \%$ were obtained, when using spermatozoa obtained through PESA for accomplishment of ICSI (3).

According to Dohle et al. (4), PESA was introduced as a less invasive technique for spermatozoa retrieval, which can be accomplished in an outpatient basis with the use of local anesthesia. The percentage of success (obtainment of motile spermatozoa) with this work was of $62 \%$ from total cases. According to the same authors, other advantages of PESA in relation to MESA are minimal discomfort for the patient, and lesser complication rate when compared to open surgery. Also, PESA does not need microsurgical instruments, like microscope, and is a very simple technique with low cost (7). According to Friedler et al. (3), after ICSI procedure, which requires the use of some spermatozoa only, cryopreservation of remaining material is feasible for the majority of patients, avoiding the necessity of future spermatozoa retrieval. In this same work, Friedler et al. accomplished the freezing of remaining PESA material, obtaining motile spermatozoa after thawing (3). Therefore, the possibility of cryopreservation for spermatozoa retrieved through PESA would be a feasible alternative.

Because PESA technique has been recently developed, there is still a little apprehension in relation to the damages it can cause to the system of ducts in the epididymis, leaving doubts in relation to the possibility of repeated procedures (8). Rosenlund et al. (8) demonstrated that PESA procedure could be successfully repeated without harming the epididymis, but there is no consensus in relation to the number of repeated procedures that can be accomplished without risks for the patient, yet.

The objective of this work is the presentation of 79 PESA procedures, analyzing the reproducibility of the technique in the same patient, and the possibility of cryopreservation of spermatozoa obtained for future use in ICSI.

\section{MATERIALS AND METHODS}

Seventy-nine PESA procedures were performed in 58 patients between August 1996 and De- cember 2000. The mean age of men was 45 years, and the mean age of their wives was 34 years. The main cause of infertility was vasectomy in 46 cases (79\%), vas deferens agenesis in 6 patients $(10 \%)$, and other causes in 6 patients (10\% - obstruction of male genital tract and failure in vasectomy reversion).

When no motile spermatozoa were found in the samples retrieved from the epididymis (negative PESA), testicular sperm aspiration (TESA) was accomplished.

\section{Study Design}

Repeated PESA procedures were performed should the patient obtain a negative result (absence of spermatozoa), in case of abortion, or desire for a second child. The material collected with PESA was used for ICSI, and the exceeding material was cryopreserved in case the patient authorized it (Figure-1). TESA was accomplished when no spermatozoa were found during epididymal puncturing.

\section{Percutaneous Epididymal Sperm Aspiration (PESA)}

All procedures have been done with local anesthesia (1\% of lidocaine for blocking the spermatic cord). Aspiration of epididymis has been done with a $27.5 \mathrm{G}$ needle coupled to a tuberculin syringe containing $0.05 \mathrm{ml}$ of Dulbecco's medium (Gibco). The epididymis was held between the surgeon's thumb and index finger. The needle was introduced in the epididymis head, the closest to the testicle (6). In each side, 2 to 3 punctures were accomplished, always changing syringes and needles for each puncture. The samples were analyzed in optical microscope to verify the presence of spermatozoa. Counting of spermatozoa was not accomplished due to the small quantity of material available. The material was store at $37^{\circ} \mathrm{C}$ up to the procedure

\section{Testicular Sperm Aspiration (TESA)}

The procedure was accomplished through local anesthesia ( $5 \mathrm{ml}$ of lidocaine for blocking the spermatic cord). The testicle was firmly held and a $16 \mathrm{G}$ needle adapted to a $20 \mathrm{ml}$ syringe filled with approxi- 


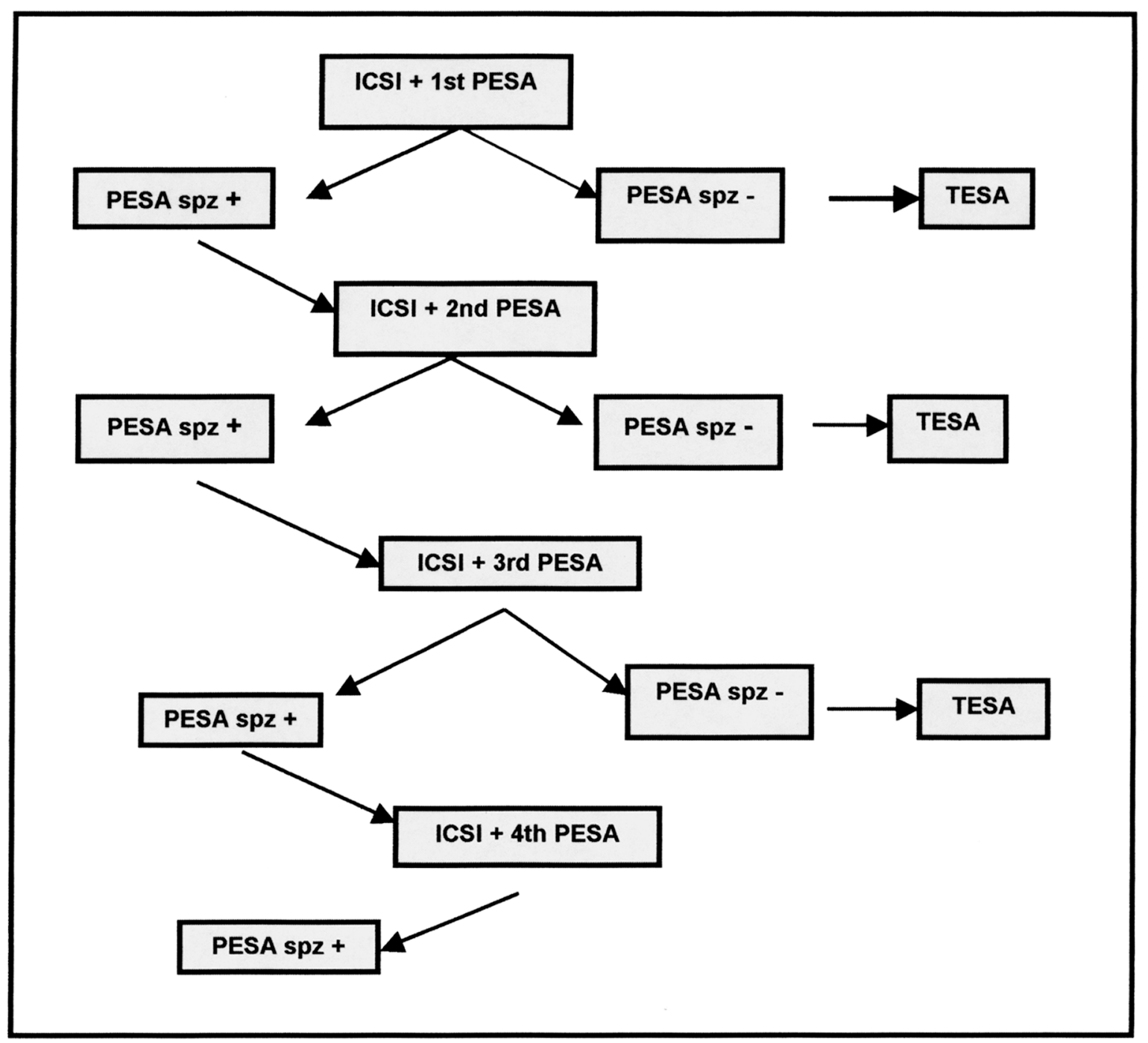

Figure 1 - Plan of retrospective study. ICSI = intracytoplasmic sperm injection, PESA = percutaneous epididymal sperm aspiration, $T E S A=$ testicular sperm aspiration, spz = spermatozoa .

mately $0.5 \mathrm{ml}$ of culture medium (modified HTF-Irvine Scientific) was introduced in the superior pole of the testicle. The aspiration was accomplished along the longitudinal axis, avoiding the epididymis head. When the needle was inside the testicular parenchyma, a negative pressure was applied to the syringe, by pulling the embolus up to $20 \mathrm{ml}$. The material collected was analyzed under optical microscope, and dissected with the help of 2 bistouries to liberate the spermatozoa from the tissue. Counting of spermatozoa was not accomplished due to the small quantity of material available.
Each testicle was punctured just once. TESA has been accomplished in 11 patients, for whom motile spermatozoa were not found with PESA.

\section{Intracytoplasmic Sperm Injection (ICSI)}

Blocking of ovarian function was accomplished with LH-RH analog followed by controlled ovarian hyper-stimulation induced through the administration of human menopausal gonadotrophin, or urofollitropin (FSH), or recombinant FSH. When a follicle reached $22 \mathrm{~mm}$, or 2 follicles reached $18 \mathrm{~mm}$ 
of maximum diameter, the hCG was intramuscularly administered. After 34-36 hours, the follicles were aspired with the help of transvaginal ultrasound. The follicular liquid was collected in a close system, in $15 \mathrm{ml}$ tubes containing $2 \mathrm{ml}$ of buffered saline solution (Dulbecco's - Gibco) added with heparin and maintained at $37^{\circ} \mathrm{C}$.

Micromanipulation was accomplished in plastic Petri dishes (Falcon, USA) with micro drops of $5 \mu \mathrm{l}$ of modified HTF medium (Irvine Scientific, USA), supplemented with $5 \%$ of human serum albumin (Irvine Scientific, USA) (9).

\section{Cryopreservation of Exceeding Material}

Part of retrieved material was used for ICSI procedure, and the remaining part was cryopreserved. With the help of a syringe or sterile graduated pipette, sample volume was measured to calculate the quantity of freezing medium to be used. Freezing medium has been used at room temperature, and carefully added with the help of sterile syringe and needle. The semen has been introduced in identified tubes of $0.5 \mathrm{ml}$. The tubes were then maintained for $10 \mathrm{~min}-$ utes under $\mathrm{N}_{2}$ vapor, and next, were dipped in liquid $\mathrm{N}_{2}$ and stored in containers up to the moment they were used.

\section{RESULTS}

PESA procedure was first accomplished with 58 patients. In 47 patients $(81 \%)$ motile spermatozoa were found (Table-1). To the 11 remaining patients (19\%) it was offered as alternative the TESA proce- dure. In all TESA procedures, it was obtained motile spermatozoa. With the spermatozoa of 58 patients, 22 pregnancies were generated (38\%), being $15(68 \%)$ from PESA spermatozoa and $7(32 \%)$ from TESA. In the first group (PESA), 7 pregnancies (47\%) were carried in full term, 7 patients suffered miscarriage, and one pregnancy was ectopic. In the second group (TESA), 5 pregnancies (71\%) were carried in full term, and 2 miscarriages occurred.

A second PESA procedure was performed in 15 patients (26\%) after a mean of 7 months from the first procedure (Table-1). Eleven couples opted to make a new attempt, for they did not obtain pregnancy in the first one. In 2 cases gestation did not succeed in full term with the first procedure, and in other 2 cases the couples are trying a second child. From these 15 procedures, in 13 (87\%) it was found motile spermatozoa. The 2 remaining patients opted for a TESA procedure to obtain spermatozoa, and both were well succeeded. In the PESA group, one gestation was carried in full term, and in the TESA group one gestation was ectopic.

A third procedure was accomplished in 5 patients (33\%) after 13.8 months from the second procedure (Table-1). Three couples opted for a new procedure because they did not obtain pregnancy in the second attempt, 1 couple due to miscarriage, and another because they were trying a second child. In 4 cases $(80 \%)$, motile spermatozoa were obtained, and only in one case it was not possible; the patient opted for TESA then. Only in the PESA group, 2 gestations were obtained, but one resulted in miscarriage. With just 1 patient a fourth procedure was performed (af-

Table 1 - Number of ICSI (intracytoplasmic sperm injection) cycles with spermatozoa coming from PESA (percutaneous epididymal sperm aspiration) or TESA (testicular sperm aspiration) and number of procedures for the same patient.

\begin{tabular}{cccc}
\hline $\begin{array}{c}\text { Number of PESA } \\
\text { procedures in the } \\
\text { same patient }\end{array}$ & Total of ICSI cycles & $\begin{array}{c}\text { ICSI cycles with } \\
\text { motile spermatozoa } \\
\text { from PESA }\end{array}$ & $\begin{array}{c}\text { ICSI cycles with motile } \\
\text { spermatozoa from TESA } \\
\text { after negative PESA }\end{array}$ \\
\hline 1 & 58 & 47 & 11 \\
2 & 15 & 13 & 2 \\
3 & 5 & 4 & 0 \\
4 & 1 & 1 & 14 \\
\hline Total & 79 & 65 & 0 \\
\hline
\end{tabular}


ter 3 months from the third one), and motile spermatozoa were found (Table-1).

A total of 79 PESA procedures were performed, and in 65 of them (82\%), motile spermatozoa were found. For patients to whom no spermatozoa were found, 14 TESA procedures were accomplished (Table-1).

Intracytoplasmic sperm injection (ICSI) has been accomplished in 587 oocytes with PESA spermatozoa, and in 153 oocytes with TESA spermatozoa. Fertilization rate was of $67 \%$ (397 among 587) for PESA cases, and 61\% (94 among 153) for TESA cases. In the PESA group, a total of 174 embryos were transferred (2.2 embryos per patient), and in the TESA group, 60 embryos (4.2 embryos per patient) were transferred. A total of 30 clinical pregnancies (presence of gestational sac) originated from 55 embryo transfers, with a gestation rate per transference of 54\% (30 among 55), being 21 gestations (38\%) from the PESA group, and $9(16 \%)$ from the TESA group. In the PESA group 9 miscarriages and 1 ectopic pregnancy occurred, while 9 gestations were carried in full term. In the TESA group, 2 miscarriages and 1 ectopic pregnancy occurred, while 9 gestations were carried in full term.

In 30 (43\%) among the 65 PESA procedures, the quantity of spermatozoa collected was enough to allow for freezing of the material after ICSI. For 13 of these 30 cases, the cryopreserved spermatozoa were thawed to be used in a new ICSI procedure. In 12 samples, motile spermatozoa were found; in these cases, 86 oocytes were injected, 59 were fertilized, 32 embryos were transferred (2.6 embryos per patient), and 2 pregnancies (16\% of gestation per transference) were obtained. From the 30 patients that had their semen cryopreserved, 4 requested that their samples were rejected, and 13 patients did not use them yet.

\section{DISCUSSION}

The simplicity of PESA associated to the efficacy of ICSI, allows that many infertile couples have their own children, as in the case of men with obstructive azoospermia (6). Many authors are confirming the efficiency of PESA $(3,7,10)$. Our results have shown that the chance of obtaining motile spermatozoa in a first PESA procedure was $81 \%$, thus confirming the efficiency of this procedure.

Besides that, there is the possibility of cryopreserving the excess of spermatozoa retrieved through PESA for future use in ICSI, avoiding the necessity of accomplishing new procedures. Friedler et al. (3) and Cayan et al. (11) report comparable rates of fertilization, cleavage and implantation after ICSI with thawed spermatozoa from the epididymis and from masturbation. Meniru et al. reported for the first time the obtainment of similar rates of gestation by transferences of cryopreserved embryos obtained with fresh epididymal spermatozoa that were cryopreserved (10). Patrizio (12) showed that spermatozoa obtained via PESA can be successfully cryopreserved, in a way as to avoid future procedures, and showed also that the fertilization and gestational rates are similar both for fresh PESA spermatozoa as well as for cryopreserved ones. In our study, it was possible to cryopreserve spermatozoa in 30 procedures (38\%), from which $13(43 \%)$ were thawed, and in 12 of them there were motile spermatozoa. From these, 2 gestations were obtained, and 4 patients opted to despise their samples, for they would not accomplish future procedure of ICSI.

Dohle et al. (7) reported that one of the advantages of PESA is its efficiency with repeated procedures. Rosenlund et al. (8) demonstrated that in men with obstructive azoospermia, PESA can be repeated in the same epididymis side for at least 3 times, with a good chance of obtaining enough quantity of motile spermatozoa for ICSI. In almost all repeated procedures of PESA, motile spermatozoa were found, including a patient who suffered 4 punctures. These results support the evidence that PESA can be successfully repeated.

\section{CONCLUSION}

PESA procedure could be considered as alternative method for spermatozoa retrieval, allowing for repetitions. The exceeding material can be cryopreserved for future use. This technique should be considered as feasible alternative for spermatozoa retrieval, helping men with obstructive azoospermia to have their own children. 


\section{REFERENCES}

1. Palermo G, Joris H, Devroey P, Van Steirteghem AC: Pregnancies after intracytoplasmic sperm injection of single spermatozoa into an oocyte. Lancet. 1992; 2 : 17-8.

2. Temple-Smith PD, Southwick GJ, Yates CA, Trounson AO, de Kretser DM: Human pregnancy by in vitro fertilization (IVF) using sperm aspirated from the epididymis. J in Vitro Fert Embryo Transf. 1985; 2: 119-22.

3. Friedler S, Raziel A, Soffer Y, Strassburger D, Komarowsky D, Ron-El R: The outcome of intracytoplasmic injection of fresh and cryopreserved epididymal spermatozoa from patients with obstructive azoospermia - a comparative study. Hum Reprod. 1998; 13: 1872-7.

4. Craft I, Tsirigotis M, Bennett V, Taranissi M, Khalifa Y, Hogewind G, et al.: Percutaneous epididymal sperm aspiration and intracytoplasmic sperm injection in the management of infertility due to obstructive azoospermia. Fertil Steril. 1995; 63: 1038-42.

5. Suzuki A, Kigure T, Harada T, Teramura T, Shimoda $\mathrm{N}$, Nishizawa O: Pregnancy obtained by in vitro fertilization (IVF) with epididymal spermatozoa in obstructive azoospermia: report of two cases. Hinyokika Kiyo. 1993; 39: 669-71.

6. Craft IL, Khalifa Y, Boulos A, Pelekanos M, Foster $\mathrm{C}$, Tsirigotis M: Factors influencing the outcome of in vitro fertilization with percutaneous aspirated epididymal spermatozoa and intracytoplasmic sperm injection in azoospermic men. Hum Reprod. 1995; 10: 1791-4.

7. Dohle GR, Ramos L, Pieters MH, Braat DD, Weber RF: Surgical sperm retrieval and intracytoplasmic sperm injection as treatment of obstructive azoospermia. Hum Reprod. 1998; 13:620-3.

8. Rosenlund B, Westlander G, Wood M, Lundin K, Reismer E, Hillensjo T: Sperm retrieval and fertilization in repeated percutaneous epididymal sperm aspiration. Hum Reprod. 1998; 13: 2805-7.

9. Mizrahi FE, Soares JB, Wonchockier R: Determination of embryo quality and its correlation with implantation rate in an assisted reproduction program. J Bras Reprod Assist. 1999; 3: 14-9 [in Portuguese].

10. Meniru GI, Gorgy A, Podsiadly BT, Craft IL: Results of percutaneous epididymal sperm aspiration and intracytoplasmic sperm injection in two major groups of patients with obstructive azoospermia. Hum Reprod. 1997; 12: 2443-6.

11. Cayan S, Lee D, Conaghan J, Givens CA, Ryan IP, Schriock ED, et al.: A comparison o ICSI outcomes with fresh and cryopreserved epididymal spermatozoa from the same couples. Hum Reprod. 2001; 16(3):495-9.

12. Patrizio P: Cryopreservation of epididymal sperm. Mol Cell Endocrinol 27. 2000; 169:11-4.

Received: May 14, 2002 Accepted after revision: February 11, 2003

$\overline{\text { Correspondance address: }}$

Dr. Sidney Glina

Rua Alte Pereira Guimarães, 360

São Paulo, SP, 01250-000, Brazil

Fax: + 5511 3747-2705

E-mail: glinas@originet.com.br 